\title{
Covid-19 e trabalhadores de enfermagem a trajetória de afastamento e retorno às atividades laborais
}

\author{
Covid-19 and nursing workers the trajectory of absence and return to work activities \\ Covid-19 y los trabajadores de enfermería la trayectoria de las actividades de baja y vuelta al \\ trabajo
}

Recebido: 29/11/2021 | Revisado: 07/12/2021 | Aceito: 12/12/2021 | Publicado: 20/12/2021

Lucas Lima de Moraes

ORCID: https://orcid.org/0000-0003-1613-5068

Universidade Estadual de Londrina, Brasil

E-mail:lucaslima16@outlook.com

Giovana Florencio

ORCID: https://orcid.org/0000-0002-5444-3101

Universidade Estadual de Londrina, Brasil

E-mail:giovana.florencio@uel.br

Dêmely Biason Ferreira

ORCID: https://orcid.org/0000-0003-1591-1213

Universidade Estadual de Londrina, Brasil

E-mail: demelybf@gmail.com

Josiane dos Santos Redon

ORCID: https://orcid.org/0000-0001-9285-684X

Universidade Estadual de Londrina, Brasil

E-mail: josiredon.enf@gmail.com

Andréia Bendine Gastaldi

ORCID: https://orcid.org/0000-0002-4081-993X

Universidade Estadual de Londrina, Brasil

E-mail: gastaldi@uel.br

Renata Perfeito Ribeiro

ORCID: https://orcid.org/0000-0002-7821-9980 Universidade Estadual de Londrina, Brasil E-mail: perfeitorenata@gmail.com

Benedita Gonçales de Assis Ribeiro

ORCID: https://orcid.org/0000-0001-5587-1098

Universidade Estadual de Londrina, Brasil E-mail: tinha@uel.br

\begin{abstract}
Resumo
Objetivo: Desvelar os sentimentos despertados no momento do diagnóstico ao processo de reintegração laboral de trabalhadores de enfermagem contaminados pela Covid-19. Metodologia: Estudo qualitativo, descritivo, que utilizou como referencial metodológico o Discurso do Sujeito Coletivo (DSC) e como referencial teórico a Teoria das Representações Sociais. A coleta de dados deu-se por entrevista semiestruturada audiogravadas abordando os participantes quanto aos seus sentimentos durante o diagnóstico de Covid-19; danos provocados pela Covid-19 em sua saúde e redes de apoio que auxiliaram no retorno às atividades laborais. Foram entrevistados 14 profissionais de saúde de um hospital referência para atendimento da Covid-19. Resultados: Em relação ao sexo, 85,7\% dos entrevistados são do sexo feminino e a mediana das idades em anos foi de 36 anos aproximadamente. Destes profissionais 76,6\% declararam-se brancos e 21,4\% pardos. Dos profissionais entrevistados, $50 \%$ são enfermeiros e $50 \%$ são técnicos de enfermagem, dos enfermeiros 28,6\% realizou pós-graduação. Os DCS são permeados pelos sentimentos de medo, ansiedade, esperança e insegurança. Todos esses, relacionados ao momento do diagnóstico até o retorno das atividades laborais. Durante o retorno as atividades laborais os profissionais relatam danos como redução da memorização que impactam na prática profissional. Considerações Finais: Sentimentos de exaustão, ansiedade, medo, insegurança, dentre outros, permearam o retorno às atividades laborais e a trajetória profissional durante a condição mundial de pandemia, as respostas a estes sentimentos podem refletir diretamente na prática e na saúde mental dos profissionais de enfermagem.
\end{abstract}

Palavras-chave: Riscos ocupacionais; Pessoal de saúde; Enfermagem; Saúde do trabalhador; COVID-19.

\section{Abstract}

Objective: To unveil the feelings aroused at the time of diagnosis to the labor reintegration process of nursing workers contaminated by Covid-19. Methods: Qualitative, descriptive study, which used as methodological reference the 
Discourse of the Collective Subject (DSC) and as theoretical reference the Theory of Social Representations. Data collection was done through semi-structured audio-recorded interviews addressing the participants about their feelings during the diagnosis of Covid-19; damage caused by Covid-19 in their health and support networks that helped them to return to work activities. Fourteen health professionals from a reference hospital for Covid-19 care were interviewed. Results: Regarding gender, $85.7 \%$ of the interviewees were female and the median age in years was 36 years old approximately. Of these professionals $76.6 \%$ declared themselves white and $21.4 \%$ brown. Of the interviewed professionals, $50 \%$ are nurses and $50 \%$ are nursing technicians. $28.6 \%$ of the nurses had a graduate degree. The DCS are permeated by feelings of fear, anxiety, hope and insecurity. All these feelings are related from the moment of diagnosis to the return to work activities. During the return to work activities the professionals report damages such as reduced memorization that impact the professional practice. Final Considerations: Feelings of exhaustion, anxiety, fear, insecurity, among others, permeated the return to work activities and the professional trajectory during the global pandemic condition; the responses to these feelings can reflect directly on the practice and mental health of nursing professionals.

Keywords: Occupational risks; Healthcare personnel; Nursing; Occupational health; COVID-19.

\begin{abstract}
Resumen
Objetivo: Desvelar los sentimientos despertados en el momento del diagnóstico al proceso de reintegración laboral de los enfermeros contaminados por la Covid-19. Metodología: Estudio cualitativo, descriptivo, que utilizó como referente metodológico el Discurso del Sujeto Coletivo (DSC) y como referente teórico la Teoría de las Representaciones Sociales. La recopilación de datos se realiza mediante entrevistas semiestructuradas y grabadas que abordan los sentimientos de los participantes durante el diagnóstico de Covid-19; los daños provocados por Covid-19 en su salud y las redes de apoyo que les ayudan a volver a sus actividades laborales. Fueron entrevistados 14 profesionales de la salud de un hospital de referencia para la atención de la Covid-19. Resultados: En relación con el sexo, el $85,7 \%$ de los entrevistados son de sexo femenino y la media de edad es de 36 años aproximadamente. De estos profesionales, el 76,6\% se declaran blancos y el 21,4\% morenos. De los profesionales entrevistados, el 50\% son enfermeros y el $50 \%$ son técnicos de enfermería. El 28,6\% de los enfermeros tienen un título de postgrado. Los DCS están impregnados de sentimientos de miedo, ansiedad, esperanza e inseguridad. Todos estos sentimientos están relacionados desde el momento del diagnóstico hasta la vuelta a las actividades laborales. Durante las actividades de reincorporación al trabajo, los profesionales informan de daños como la reducción de la memorización que repercuten en la práctica profesional. Consideraciones finales: Los sentimientos de agotamiento, ansiedad, miedo, inseguridad, entre otros, impregnaron las actividades de retorno al trabajo y la trayectoria profesional durante la condición de pandemia global; las respuestas a estos sentimientos pueden reflejarse directamente en la práctica y la salud mental de los profesionales de enfermería.
\end{abstract}

Palabras clave: Riesgos laborales; Personal de salud; Enfermería; Salud laboral; COVID-19.

\title{
1. Introdução
}

O SARS-CoV-2 é um vírus com Ácido Ribonucleico (RNA) da família dos coronavírus que provoca uma síndrome respiratória grave em humanos. Em decorrência da alta transmissibilidade desta nova variante do coronavírus, no fim de 2019, o mundo se inseriu em uma situação emergencial declarada pela Organização Mundial de Saúde (OMS) como uma emergência de saúde pública de importância internacional (Yesudhas et al., 2020; WHO, 2019).

De dezembro de 2019 a julho de 2021 a doença foi responsável por mais de 185 milhões de contaminados e mais de 4 milhões de mortes, no mundo. No Brasil os dados estatísticos demonstram o avanço exacerbado da contaminação e do número de mortes, atualmente, sendo responsável por mais de 18 milhões de casos confirmados da Covid-19 e mais de 520 mil óbitos (WHO, 2021).

Informações acerca da transmissibilidade, fisiopatologia e sintomatologia são melhores elucidados pela literatura dado ao avanço dos estudos nesta área, mostrando que as complicações mais graves são mais frequentes em idosos e indivíduos com comorbidades como hipertensão, diabetes mellitus, doença cardiovascular, doença pulmonar crônica e câncer. Associa-se a Covid-19 à dispneia, hipoxemia, insuficiência respiratória, choque e insuficiência de múltiplos órgãos (Chams et al., 2020; Wu \& McGoogan, 2020). Os sintomas mais comuns em pacientes com diagnóstico de Covid-19 são: febre, tosse, mialgia ou fadiga; e sintomas atípicos como: escarro, cefaleia, hemoptise e diarreia (Huang et al., 2020).

Dada a alta transmissibilidade e o número crescente de pacientes contaminados que necessitavam de internação, os profissionais de saúde foram designados a enfrentar um grande desafio: o de cuidar de si e dos outros se expondo ao contágio 
(Restrepo-Martínez et al., 2021). Portanto, em sua rotina passaram a se deparar com um número cada vez maior de pacientes gerando superlotação dos hospitais, inúmeros casos que evoluíram a óbito, escassez de recursos materiais, poucas informações acerca da doença e, principalmente, o risco de se infectar durante sua jornada de trabalho. Logo, sintomas relacionados a ansiedade e depressão tornaram-se mais frequentes entre esses trabalhadores, impactando diretamente na saúde dos mesmos (Pereira et al., 2021).

Estudos realizados com profissionais de saúde que vivenciaram outro surto de coronavírus na China em 2003 demonstraram que os profissionais de saúde estiveram mais propensos a desenvolver problemas de saúde mental, bem como, estresse pós-traumático. Entrelaçado a está questão esses profissionais que atuaram durante o surto em 2003 relataram sofrer com ansiedade, frustração e depressão (Xiang et al., 2020). De acordo com Moreira WC et. al (2020) durante a pandemia da Covid-19 os transtornos de ansiedade aumentaram gerando repercussões psicológicas negativas nas pessoas que tem contato direto com tal realidade, em especial, na classe dos trabalhadores de saúde.

Dados revelados pelo Ministério da Saúde, revelou que mais de 173 mil profissionais de saúde foram contaminados pela Covid-19 e que a maior parte deles são profissionais de Enfermagem, representando mais de 49\% dos casos (Brasil, 2020). A equipe de Enfermagem representa a maior parcela de profissionais nas instituições de saúde. Logo estes profissionais estão mais expostos e mais vulneráveis à contaminação.

Dado o exposto, compreender os sentimentos dos profissionais de enfermagem que foram contaminados pela Covid19 é de extrema importância para que as instituições possam planejar estratégias que auxiliem esses trabalhadores no retorno das atividades laborais com intuito de prevenir e auxiliar nos processos de adoecimento mental, logo o objetivo deste estudo foi desvelar os sentimentos despertados do momento do diagnóstico ao processo de reintegração ao trabalho de profissionais de enfermagem contaminados pela Covid-19.

\section{Metodologia}

Trata-se de um estudo qualitativo realizado em um Hospital terciário do Norte do Paraná, referência para o atendimento de pacientes com Covid-19. Os profissionais de enfermagem que passaram por tratamento da Covid-19, foram convidados durante seu turno de trabalho a participar do estudo. Participaram 14 trabalhadores, que assinaram o Termo de Consentimento Livre e Esclarecido (TCLE).

A coleta de dados foi realizada no período de fevereiro de 2021 a junho de 2021, empregando-se as técnicas investigativas de entrevista semi-estruturada e observação dos participantes. A entrevista foi gravada, utilizando o gravador de áudio como um instrumento de auxílio no registro. Com intuito de garantir o sigilo dos participantes no início da entrevista o pesquisador entregou crachás com nomes fictícios e não foram divulgadas informações de caráter pessoal dos entrevistados. Posteriormente as entrevistas foram transcritas na integra pelo pesquisador e os áudios foram deletados. Os dados sociodemográficos foram coletados por meio de um questionário previamente elaborado pelos pesquisadores.

Durante as entrevistas os participantes foram questionados quanto aos seus sentimentos durante o diagnóstico de Covid-19; danos provocados pela Covid-19 em sua saúde; e redes de apoio que auxiliaram no retorno às atividades laborais. O critério adotado para determinar o tamanho amostral foi a saturação dos dados, que pode ser compreendido como o momento em que a busca de novos sujeitos não acrescenta novas informações (Victoria et al., 2000).

O referencial teórico que permeou este estudo foi a Teoria das Representações sociais. Está teoria têm por fundamento principal o poder das ideias, assim parte do princípio de que as pessoas partilham ideias e consequentemente transformam suas ideias em práticas. Os novos fenômenos, resultado das ideias em prática, são compreendidos sob a ótica pscicossociológica e criativa (Moscovici, 2013). 
$\mathrm{O}$ autor refere que sua teoria é reflexo de uma sociedade pensante. O paradigma por ele utilizado é dinâmico, o qual vai ao encontro as explicações de mudanças e inovações sociais, contrário à manutenção fixa de uma visão de mundo (Sá, 1955).

A técnica utilizada para organizar e analisar os dados obtidos por meio das entrevistas foi o DSC, trata-se de uma metodologia que busca desvelar as representações sociais com características qualitativas e com possibilidade de associá-la a dados quantitativos (Lefevre \& Lefevre, 2014).

A técnica de análise DSC consiste em etapas que buscam extrair do conteúdo as Ideias Centrais (IC); Expressões chaves (ECH); Ancoragem (AC) e o DSC. Este DSC que, por fim, desvelará as representações sociais que tem por característica a fala de um coletivo por meio de um sujeito, único, falado (Lefevre \& Lefevre, 2014).

As IC são compreendidas como uma expressão linguística que descreve e nomeia, de forma sintética, os sentidos presentes em cada uma das respostas analisadas e de cada ECH. As ECH são trechos dos discursos individuais que revelam a essência do conteúdo dos discursos, e são extraídas da matéria prima pelo pesquisador. As AC são enunciados que contém uma teoria, uma crença, que é professada pelo sujeito (Figueiredo et al., 2014; Lefevre \& Lefevre, 2014).

Assim, as entrevistas foram analisadas seguindo as etapas propostas pelo DSC. O primeiro passo executado, foi a transcrição das entrevistas na integra em uma coluna denominada ECH. Posteriormente, no segundo momento, identificamos em cada resposta, as ECH das IC e excluímos o discurso excedente. Em um terceiro momento identificamos e descremos as IC e ECH. No quarto momento realizamos o agrupamento das IC com o mesmo sentido e enumeramos em algarismos romanos I, II, III, IV e assim por diante. Para cada agrupamento criamos uma IC síntese que expresse melhor um conjunto de IC e AC. No último momento realizamos a construção dos DSC que serão apresentados nos resultados.

O presente estudo pertence a dissertação intitulada "Trabalhadores dos Serviços de saúde Frente à pandemia de COVID-19" aprovado pelo Comitê de Ética em Pesquisas com Seres Humanos da Universidade Estadual de Londrina-PR sob número de parecer 5125297.

\section{Resultados}

\subsection{Dados sociodemográficos e características relacionadas ao trabalho}

Participaram deste estudo 14 profissionais de enfermagem, onde $85,7 \%$ dos entrevistados são do sexo feminino, a mediana das idades em anos foi de 36 anos aproximadamente. Destes profissionais $76,6 \%$ se declararam brancos e $21,4 \%$ pardos.

Dos profissionais entrevistados, $50 \%$ são enfermeiros e 50\% são técnicos de enfermagem e entre os enfermeiros, 28,6\% realizou pós-graduação. A mediana do tempo de formação em anos é de aproximadamente nove anos e do tempo de atuação na área é de oito anos, além de que, 64,3\% dos profissionais entrevistados possuem outro vínculo empregatício com a mesma função e $14,3 \%$ dos profissionais realizam hora extra nesta instituição, tendo como média de 36 horas semanais trabalhadas.

3.2 Os sentimentos despertados ao receber o diagnóstico de Covid-19, danos provocados a saúde e processo de retorno as atividades laborais.

Ideia Central A- Os principais sentimentos despertados ao receber o diagnóstico de Covid-19 são o medo e a ansiedade e eles estão relacionados ao agravamento do quadro, a transmissão da doença e a morte.

Medo, medo de sentir algo pior e se agravar. Eu estou com a doença, eu estou contaminado e como vai ser daqui para frente? Como eu vou desenvolver a doença no passar dos dias? Será que eu não vou ter agravamento do 
quadro? Será que eu vou ficar internada? A gente vê muita gente com Covid, já vi muita gente morrer. Vem o sentimento, também, de medo de você passar para os familiares, fico preocupada em passar para minha família? (DSC-A).

Nesse discurso podemos perceber que o cuidar de pacientes acometidos pela Covid-19 é um fator desencadeador de ansiedade e medo nos profissionais que atendem na linha de frente e foram contaminados. A incerteza gerada pela pandemia do novo coronavírus também contribui para despertar os sentimentos nos profissionais.

Ideia Central B- O cansaço persistente, redução de memória e principalmente perda de olfato e paladar impactam diretamente na qualidade de vida e no trabalho.

Fica com muitos sintomas, principalmente o cansaço. Quando eu retornei ao trabalho eu via que depois de um esforço eu ficava fadigada aí acabei percebendo, antes eu não tinha hoje eu tenho. Demora para recuperar, mas diminui o cansaço. Percebi também que minha memória e visão ficou diferente. Até hoje meu olfato não voltou e isso é uma coisa que atrapalha (DSC - B)

Nesse discurso percebe-se que os profissionais que foram contaminados pelo novo coronavírus reconhecem danos provocados em sua saúde sendo os principais elencados o cansaço, perda de olfato e dificuldade visual e de memória quanto a persistência destes sintomas é possível afirmar que em alguns trabalhadores os danos foram temporários, porém em outros persistentes.

Ideia Central $C$ - $O$ medo de contrair a doença novamente está entrelaçado ao alívio de retornar ao trabalho no fim do isolamento, estes são os principais sentimentos.

Da um certo medo eu ficava me questionando: e se eu ainda estou transmitindo? E se eu pegar novamente? Medo de contrair novamente e ficar pior ter um caso mais grave. Eu via os pacientes novos com Covid e pensava: E se eu pegar novamente? Mas fiquei feliz e aliviada em retornar saber que eu venci e estou de volta a luta (DSC-C).

Ao fim do isolamento o profissional revela que os principais sentimentos são o medo e alívio, mesmo com a necessidade de retornar ao possível cenário que o contaminou. Para profissional o fim do isolamento representa um momento como uma vitória sem grandes danos.

Ideia Central D-A ansiedade e insegurança estão presentes no momento da reintegração e retorno das atividades laborais, porém o acolhimento dos colegas de trabalho é um fator positivo para a reintegração

Quando eu cheguei na frente do hospital eu chorava, chorava... não tinha condições de trabalhar, chorei muito e tive crise de pânico. Eu me sentia insegura em cuidar dos pacientes tão graves, paciente com Covid... Mas eu senti muito apoio do pessoal, todo mundo querendo saber como eu estava. Várias pessoas me mandaram mensagem. Eu senti que as pessoas se importavam comigo, me senti querida (DSC-D). 
Entendemos que está ideia central D desvela as representações do sentimento de medo e de pânico quando o profissional necessita entrar no cenário, contrário ao sentimento de alívio desvelado no momento do fim do isolamento. Porém o acolhimento dos colegas de trabalhos para com os funcionários que estão retornando do período de isolamento é um fator positivo para que se obtenha o êxito, os entrevistados revelam que sentir-se "querido, importante" fortalecem o retorno.

\section{Ideia Central E- As redes de apoio como colegas de trabalho e chefia contribuem para o sucesso no retorno das atividades, mas sente-se falta de acompanhamento.}

Minha chefe perguntou se eu estava bem e se conseguia retornar para mesma atividade, nos primeiros dias os colegas me ajudavam pra eu não cansar tanto. Minha chefe também perguntou todos os dias como eu estava. $O$ médico me disse que os sintomas eram assim mesmo e que iriam passar, aí eu fiquei mais tranquila. Depois que passou o isolamento não tive um acompanhamento (DSC-E).

Entendemos que a ideia central E aborda grandes representações no que tange a importância da chefia junto ao profissional, percebe-se um sentimento de segurança advindo desta rede de apoio que junto aos colegas de trabalho alicerçam um fator positivo para reintegração. Os trabalhadores revelam que os serviços de apoio existem, mas não tiveram maiores informações de como acessá-los.

\section{Discussão}

A condição mundial de pandemia desvelou uma característica histórica dos profissionais de enfermagem a desvalorização e necessidade de dupla jornada de trabalho (Rosa et al., 2021). A necessidade de mão de obra e de profissionais capacitados em cuidar têm impulsionado a dupla jornada e carga horária excessiva de horas extra contribuindo para a manifestação de danos à saúde destes profissionais uma vez que estão mais expostos ao agente etiológico, bem como, a fatores estressantes (Xiang et al., 2020).

O cansaço e exaustão passaram a fazer parte da rotina destes profissionais culminando assim em estresse e redução da qualidade de vida. O desconhecido, novo coronavírus, além de provocar desgaste físico nos profissionais, ocasionou desgaste emocional demonstrado pelo aumento de profissionais com ansiedade, e o medo são consequências recorrentes do trabalho em ambientes destinados ao tratamento da Covid-19 (Kang et al., 2020).

Entrelaçado ao cansaço, ansiedade e medo os profissionais passaram a se deparar com pessoas que foram contaminadas durante o trabalho, além da intensificação da sobrecarga emocional do cenário marcado pela piora clínica e óbito dos pacientes. Um estudo realizado com profissionais que cuidam de pacientes com Covid-19 demonstrou que o medo e ansiedade, frequentemente, estão relacionados ao temor de transmitir a doença para seus familiares. (Rossi et al., 2020)

A alta transmissibilidade e o avanço da pandemia tornou esses números de contaminados mais próximos colocando assim os profissionais na condição de isolamento. Quando se compara profissionais com suspeita de Covid-19 e profissionais sem a suspeita, observa-se que o nível de ansiedade foi maior no grupo com a suspeita (Santos et al., 2021). Assim, podemos subentender que ao receber o diagnóstico de Covid-19 os níveis de ansiedade tendem a aumentar.

A projeção do cuidado é uma característica presente no cuidado profissional de enfermagem por tratar-se de uma profissão que lida diretamente com as diversas situações e demandas de saúde (Zuchetto et al., 2019). Essa característica pode desencadear processos de sofrimento mental e interferir na prática profissional. Neste estudo pode-se compreender que as projeções estão presentes, uma vez que os profissionais visualizam sua possível piora clínica baseada em vivências e experiências passadas de pessoas em que a doença cursou de forma grave. 
O envolvimento e as relações construídas com o outro são processos inerentes a profissão, uma vez que o percurso de cuidar está intimamente relacionado a convivência diária e intensa, assim as mesmas relações construídas com os que precisam do cuidado são construídas entre os profissionais. Portanto das interações emergem representações sociais reflexo de uma população que vivencia uma mesma situação.

As sequelas da Covid-19 é uma temática de suma importância, uma vez que afetam diretamente a qualidade de vida, estudos publicados reforçam a importância da temática e a necessidade de equipes destinadas a reabilitação destas pessoas. (Iqbal et al., 2021; Kamal et al., 2020).

Diversos estudos publicados demonstraram e validaram o impacto da pandemia da Covid-19 na saúde mental dos profissionais de enfermagem (Moreira \& De Lucca, 2020; Medeiros Lima et al., 2020; Prado et al., 2020). Achados que corroboram com nosso estudo uma vez que os participantes referem como principais danos à saúde percebidos a perda de olfato e paladar, redução da capacidade de memorização e fadiga.

Em consonância os dados supracitados nosso estudo demonstra que a ansiedade e o medo durante o retorno das atividades laborais são representações social da população estudada. Ao retornar ao trabalho a maior representatividade evidenciada está correlacionada ao fato de retornar e cuidar de pessoas com Covid-19.

Quando investigado os fatores que subsidiaram o retorno a prática da empatia é a principal habilidade social observada por meio dos relatos que propiciaram aos profissionais um melhor retorno. resposta das interações sociais. Logo a empatia pode ser compreendida como a capacidade de identificar, compreender e experimentar os sentimentos do outro (Del Prette \& Del Prette, 2017).

Assim, a empatia é uma ferramenta que pode transformar as relações interpessoais quando utilizada no processo de acolhimento (Savieto \& Leão, 2016). O acolhimento está intimamente relacionado a empatia e a partir dos dados levantados observa-se inferências indiretas da empatia no processo de acolhimento dos profissionais de enfermagem que retornaram as suas atividades laborais

Portanto, reforçar redes de apoio e a interação de ajuda entre os profissionais, é de suma importância (RamosToescher et al., 2020), percebendo-se que os profissionais quando retornaram ao labor, perceberam o acolhimento como uma rede de apoio, sendo dessa forma, um fator positivo para o retorno destes profissionais.

Compreendendo que as relações sociais geram representações sociais e essas são baseadas em comunicações entre a população que está exposta a uma mesma realidade, os dados acima discutidos podem demonstrar as principais características que representam esses profissionais que foram contaminados pela Covid-19 e precisaram retornar as atividades laborais.

\section{Considerações Finais}

Os resultados deste estudo buscaram desvelar os principais sentimentos/emoções vivenciadas por profissionais de enfermagem que atuam na linha de frente durante a pandemia da COVID-19 e que foram contaminados e retornaram as atividades laborais.

Sentimentos de exaustão, ansiedade, medo, insegurança, dentre outros permearam o retorno às atividades laborais e a trajetória profissional durante a condição mundial de pandemia que podem resultar em grandes danos à saúde mental destes profissionais. Buscou-se refletir à luz da teoria das representações sociais por meio do discurso do sujeito coletivo por tratar-se de referenciais teórico e metodológico que subsidiam as investigações acerca das interações sociais.

Espera-se que os dados revelados por este estudo instiguem a elaboração de estudos investigando os danos à saúde destes trabalhadores devem ser realizados para que se possa criar estratégias eficazes e assertivas no que tange a reintegração dos trabalhadores e o processo de trabalho durante momentos de pandemia. Ainda, espera-se que este estudo possa subsidiar 
ações que fortaleçam a prática dos profissionais de enfermagem e as futuras ações de apoio aos profissionais que receberam o diagnóstico de Covid-19.

\section{Referências}

Amorin Zuchetto, M., Engel, F. D., Pacheco de Medeiros, L. S., Silveira de Almeida Hammerschmidt, K., \& Dornelles Schoeller, S. (2019). Empatía en el proceso de cuidado en enfermería bajo la óptica de la teoría del reconocimiento: Síntesis reflexiva. Revista Cuidarte, 10(3). https://doi.org/10.15649/cuidarte.v10i3.624

Brasil. Boletim epidemiológico da COVID 19 balanço de infecções dos profissionais de saúde. 2020. https://www.saude.gov.br/noticias/agencia-saude/47179novo-boletim-epidemiologico-da-covid-19-traz-balanco-de-infeccoes-em-profissionais-de-saude

Chams, N., Chams, S., Badran, R., Shams, A., Araji, A., Raad, M., Mukhopadhyay, S., Stroberg, E., Duval, E. J., Barton, L. M., \& Hajj Hussein, I. (2020). COVID-19: A multidisciplinary review. Frontiers in Public Health, 8. https://doi.org/10.3389/fpubh.2020.00383

Figueredo, M.Z.A; Chiari, B.M; Goulart, B.N.G. Discurso do Sujeito Coletivo: uma breve introdução à ferramenta de pesquisa qualiquantitativa. (2013). Distúrbios da comunicação, 25(1).

Huang, C., Wang, Y., Li, X., Ren, L., Zhao, J., Hu, Y., Zhang, L., Fan, G., Xu, J., Gu, X., Cheng, Z., Yu, T., Xia, J., Wei, Y., Wu, W., Xie, X., Yin, W., Li, H., Liu, M., \& Cao, B. (2020). Clinical features of patients infected with 2019 novel coronavirus in Wuhan, China. The Lancet, 395(10223), 497-506. https://doi.org/10.1016/s0140-6736(20)30183-5

Iqbal, A., Iqbal, K., Arshad Ali, S., Azim, D., Farid, E., Baig, M. D., Bin Arif, T., \& Raza, M. (2021). The COVID-19 sequelae: A cross-sectional evaluation of post-recovery symptoms and the need for rehabilitation of COVID-19 survivors. Cureus. https://doi.org/10.7759/cureus.13080

Kamal, M., Abo Omirah, M., Hussein, A., \& Saeed, H. (2020). Assessment and characterisation of post-COVID-19 manifestations. International Journal of Clinical Practice, 75(3). https://doi.org/10.1111/ijcp.13746

Kang, L., Li, Y., Hu, S., Chen, M., Yang, C., Yang, B. X., Wang, Y., Hu, J., Lai, J., Ma, X., Chen, J., Guan, L., Wang, G., Ma, H., \& Liu, Z. (2020). The mental health of medical workers in Wuhan, China dealing with the 2019 novel coronavirus. The Lancet Psychiatry, 7(3), e14. https://doi.org/10.1016/s22150366(20)30047-x

Lefevre, F., \& Lefevre, A. M. C. (2014). Discourse of the collective subject: Social representations and communication interventions. Texto \& Contexto Enfermagem, 23(2), 502-507. https://doi.org/10.1590/0104-07072014000000014

Medeiros Lima, A., Moraes Soares Motta de Carvalho, C., Macedo Angelo, L., Alves de Oliveira, M., Pereira de Oliveira da Silva, P. C., Sousa dos Santos, R. G., \& Oliveira de Carvalho da Silva, R. (2020). Relações entre a pandemia de COVID-19 e a saúde mental dos profissionais de enfermagem. Saúde Coletiva (Barueri), 54, 2699-2706. https://doi.org/10.36489/saudecoletiva.2020v10i54p2699-2706

Moreira, A. S., \& De Lucca, S. R. (2020). Apoio psicossocial e saúde mental dos profissionais de enfermagem no combate ao covid-19. Enfermagem Em Foco, 11(1.ESP). https://doi.org/10.21675/2357-707x.2020.v11.n1.esp.3590

Moreira, W. C., Sousa, A. R. de, \& Nóbrega, M. do P. S. de S. (2020a). Mental illness in the general population and health professionals during covid-19: a scoping review. Texto \& Contexto - Enfermagem, 29. https://doi.org/10.1590/1980-265x-tce-2020-0215

Moscovici, S. (2013). Representações sociais: Investigações em psicologia social. Vozes

Prado, A. D., Peixoto, B. C., Da Silva, A. M. B., \& Scalia, L. A. M. (2020). A saúde mental dos profissionais de saúde frente à pandemia do COVID-19: Uma revisão integrativa. Revista Eletrônica Acervo Saúde, 46, e4128. https://doi.org/10.25248/reas.e4128.2020

Pereira, A. C. C., Pereira, M. M. A., Silva, B. L. L., Freitas, C. M. de, Cruz, C. S., David, D. B. M., Santos, D. L., Delfraro, D. O., \& Ura, F. A. C. (2021). O agravamento dos transtornos de ansiedade em profissionais de saúde no contexto da pandemia da COVID-19 / The aggravation of anxiety disorders in healthcare professionals in the context of COVID-19 pandemic. Brazilian Journal of Health Review, 4(2), 4094-4110. https://doi.org/10.34119/bjhrv4n2-009

Ramos-Toescher, A. M., Tomaschewisk-Barlem, J. G., Barlem, E. L. D., Castanheira, J. S., \& Toescher, R. L. (2020). Saúde mental de profissionais de enfermagem durante a pandemia de COVID-19: Recursos de apoio. Escola Anna Nery, 24(spe). https://doi.org/10.1590/2177-9465-ean-2020-0276

Restrepo-Martínez, M., Escobar, M., Marín, L. A., \& Restrepo, D. (2021). Prevalencia y características clínicas de los síntomas depresivos y ansiosos de los trabajadores de una institución de salud en Medellín durante la pandemia por COVID-19. Revista Colombiana de Psiquiatría. https://doi.org/10.1016/j.rcp.2021.02.001

Rosa, T. J. L., Nascimento, S. M., de Sousa, R. R., \& Oliveira, D. M. do N. (2021). Análise sobre a saúde mental dos profissionais de enfermagem no enfrentamento da covid-19: uma análise num hospital regional. Brazilian Journal of Development, 7(5), $44293-44317$. https://doi.org/10.34117/bjdv.v7i5.29229

Rossi, R., Socci, V., Pacitti, F., Di Lorenzo, G., Di Marco, A., Siracusano, A., \& Rossi, A. (2020). Mental health outcomes among frontline and second-line health care workers during the coronavirus disease 2019 (COVID-19) pandemic in italy. JAMA Network Open, 3(5), e2010185. https://doi.org/10.1001/jamanetworkopen.2020.10185

SÁ, C. P. de. Representações Sociais: o conceito e o estado atual da teoria. In: SPINK, M. J. (org.). O conhecimento no cotidiano. Brasiliense, 1995, 1957.

Santos, K. M. R. dos, Galvão, M. H. R., Gomes, S. M., Souza, T. A. de, Medeiros, A. de A., \& Barbosa, I. R. (2021). Depressão e ansiedade em profissionais de enfermagem durante a pandemia da covid-19. Escola Anna Nery, 25(spe). https://doi.org/10.1590/2177-9465-ean-2020-0370 
Research, Society and Development, v. 10, n. 17, e37101723943, 2021

(CC BY 4.0) | ISSN 2525-3409 | DOI: http://dx.doi.org/10.33448/rsd-v10i17.23943

Victora, C. G., Knauth, D. R., \& Hassen, M. D. N. A. (2000). Pesquisa qualitativa em saúde: Uma introdução ao tema.

WHO Coronavirus disease 2019 (COVID-19) Situation Report-57.2020. https://www.who.int/docs/default-source/coronaviruse/situation-reports/20200428sitrep-99-covid-19.pdf?sfvrsn=119fc381_2

WHO World Health organization Coronavirus (COVID-19) Dashboard. 2021. Acesso em 10 de julho de 2021. https://covid19.who.int/

Wu, Z., \& McGoogan, J. M. (2020). Characteristics of and important lessons from the coronavirus disease 2019 (COVID-19) outbreak in China. JAMA, 323(13), 1239. https://doi.org/10.1001/jama.2020.2648

Yesudhas, D., Srivastava, A., \& Gromiha, M. M. (2020). COVID-19 outbreak: History, mechanism, transmission, structural studies and therapeutics. Infection, 49(2), 199-213. https://doi.org/10.1007/s15010-020-01516-2

Del Prette A. \& Del Prette Z. A. P. (2017). Competência Social e Habilidades Sociais: manual teórico-prático. Vozes

Savieto R. M. \& Leão E. R. (2016). Assistência em enfermagem e Jean Watson: uma reflexão sobre a empatia. Escola Anna Nery Revista de Enfermagem [Internet]. 20(1): 198-202. http://www.scielo.br/scielo.php?script=sci_arttext\&pid=S1414-81452016000100198\&lng=en. https://doi.org/10.5935/14148145.20160026 .

Shigemura, J., Ursano, R. J., Morganstein, J. C., Kurosawa, M., \& Benedek, D. M. (2020). Public responses to the novel 2019 coronavirus (2019-nCoV) in Japan: Mental health consequences and target populations. Psychiatry and Clinical Neurosciences, 74(4), 281-282. https://doi.org/10.1111/pcn.12988

Xiang, Y.-T., Yang, Y., Li, W., Zhang, L., Zhang, Q., Cheung, T., \& Ng, C. H. (2020). Timely mental health care for the 2019 novel coronavirus outbreak is urgently needed. The Lancet Psychiatry, 7(3), 228-229. https://doi.org/10.1016/s2215-0366(20)30046-8 